\section{Investigación-creación en Colombia: la formulación del "nuevo" modelo de medición para la producción intelectual en artes, arquitectura y diseño}

\section{Resumen}

Se presenta una reflexión alrededor del proceso y formulación participada del "nuevo" modelo de valoración de la producción e impacto de la investigación-creación de las artes, arquitectura y diseño. Esta propuesta, desarrollada por los autores de este artículo, extiende el modelo oficial de medición de la producción científica en Colombia, utilizado para clasificar investigadores y grupos de investigación. Para su desarrollo, las asociaciones de las facultades y programas de arte, arquitectura y diseño del país trabajaron de forma conjunta con entidades gubernamentales encargadas de promover la producción científica, tecnológica, creativa y cultural nacional. A través de este proceso participativo innovador como proceso de negociación entre las asociaciones y el Estado, se fundamentó epistemológicamente el tipo de conocimiento resultado de la investigación-creación, y se establecieron los requerimientos de valoración de la calidad de estos productos de forma articulada a las propias instancias de valoración de la producción creativa-cultural, tales como selecciones curadas o concursos. El nuevo modelo fruto de un proceso de negociación en la mesa de trabajo fue asumido por el estado de forma oficial, y puesto en marcha en 2015 como un piloto sin medición y luego como instrumento de medición en 2017, dando relevancia a otros sistemas de generación de conocimiento, no científicos, y valorando su impacto en la promoción de industrias creativas y culturales, así como en el fortalecimiento del Sistema Nacional de Ciencia, Tecnología e Innovación - SNCTI.
Héctor Antonio Bonilla Estévez Doctor (c) en Filosofía

Profesor Universidad Antonio Nariño

Bogotá, Colombia

Correo electrónico: excelencia.docente@uan.edu.co orcid.org/0000-0002-8476-394X

Google Scholar

Francisco Cabanzo, PhD

Doctor en Arte y Pensamiento

Profesor Universidad El Bosque

Bogotá, Colombia

Correo electrónico: acabanzo@unbosque.edu.co orcid.org0000-0001-6783-6185 Google Scholar

Tania Catalina Delgado, $\mathrm{PhD}$ Doctor in Design and Technologies Exploitation for Doctor in Design and

the Cultural Heritage Directora de Mentalidad y Cultura de Colciencias. Correo electrónico: tcdelgado@colciencias.gov.co Google Scholar

Oscar Andrés Hernández Salgar, PhD Doctor en Ciencias Sociales y Humanas
Profesor Pontificia Universidad Javeriana Bogotá, Colombia

Bogotá, Colombia
Correo electrónico: oscar.hernandez@javeriana.edu.co orcid.org/0000-0003-4041-434X Google Scholar

Alexander Stward Niño Soto, PhD Doctor en Ciudad, Territorio y Sustentabilidad Profesor Universidad del Norte, Barranquilla, Colombia

Correo electrónico: asnino@uninorte.edu.co Correo electrónico: asnino@unin Google Scholar

Juan Salamanca, PhD

Doctor in Design

Assistant professor. School of art + design University of Illinois at Urbana Champaign Correo electrónico: jsal@illinois.edu orcid.org/0000-0002-4868-171X

Google Scholar

Recibido: Febrero 27 de 2018

Aprobado: Enero 1 de 2019

\section{Palabras clave:}

Desarrollo tecnológico, innovación, investigación acción participativa, investigación-creación, valoración.

Revista KEPES Año 16 No. 20 julio-diciembre 2019, págs. 673-704 ISSN: 1794-7111(Impreso) ISSN: 2462-8115 (En línea) DOI: 10.17151/kepes.2019.16.20.24 


\section{Research-creation in Colombia: the formulation of the "new" measurement model for intellectual production in arts, architecture and design}

\begin{abstract}
A reflection about the process and participatory formulation of the "new" model for the evaluation of the production and impact of research-creation of the arts, architecture and design is presented. This proposal, developed by the authors of this paper, extends the official measurement model of scientific production in Colombia, used to classify researchers and research groups. For its development, the associations of the faculties and programs of art, architecture and design of the country worked jointly with governmental entities in charge of promoting the national scientific, technological, creative and cultural production. Through this innovative participatory process, as a negotiation process between the associations and the state, the type of knowledge resulting from researchcreation was based epistemologically, and the requirements for assessing the quality of these products were established in an articulated manner to the instances of valuation of creativecultural production, such as curated selections or contests. The new model, resulting from a negotiation process in the workgroups, was officially assumed by the state, and launched in 2015 as a pilot model without measurement and then as a measuring instrument in 2017, giving relevance to other nonscientific systems of generation of knowledge, and assessing their impact on the promotion of creative and cultural industries, as well as on strengthening the National Science, Technology and Inovation System (NSTIS).
\end{abstract}

Key words:

Evaluation, innovation, participatory action research, research-creation, technological development. 


\section{Introducción}

La Mesa Nacional de Artes, Arquitectura y Diseño (Mesa AAD) conformada por los representantes de la Asociación Colombiana de Facultades y Programas de Artes (ACOFARTES), Asociación Colombiana de Facultades de Arquitectura (ACFA) y Red Académica de Diseño (RAD) de Colombia ${ }^{1}$, por el Departamento Administrativo de Ciencia, Tecnología e Innovación, Colciencias, junto con otras entidades interesadas en reconocer el rol que tienen los procesos de investigación-creación en las facultades de esas disciplinas en la generación de nuevo conocimiento, desarrollo tecnológico e innovación del país, constituyeron desde 2013 una mesa de negociación / mesa de trabajo (mesa AAD) $)^{2}$, enfocada a construir, discutir y negociar un nuevo modelo capáz de incluir tanto productos como procesos de generación de conocimiento propios de estas disciplinas en el Sistema Nacional de Ciencia, Tecnología e Innovación - SNCTI.

En términos metodológicos, no se plantea un diseño previo a la constitución de la Mesa AAD, su desarrollo fue incremental. Al inicio el esfuerzo de los integrantes convocados por Colciencias se concentró en estudiar los modelos de reconocimiento y valoración adoptados por las propias universidades para reconocer y medir los procesos y productos de creación propios del arte, la arquitectura y el diseño (Asprilla, 2013; Hernández, 2006), esa construcción nacional se confrontó con otros modelos aplicados internacionalmente (Chile, México, Italia, Australia) se extrajeron las características fundamentales de cada subtema, tanto en el reconocimiento como en la valoración. Se hizo una reflexión acerca de las categorías descriptivas de la "existencia" de los productos, desde las diferencias y características propias de la producción artística, llegando a unos listados. La revisión de dichos listados condujo a la

\footnotetext{
30 facultades de arte de Colombia pertenecen a ACOFARTES, 37 facultades de arquitectura a ACFA y 51 programas de diseño a RAD. ${ }^{2}$ El énfasis no está en el espacio, sino en el verbo y aparece en estado transitivo: y entonces la negociación no está cerrada mientras haya temas abiertos, la negociación es entonces un estado y no un lugar (Zuzek, Talik, Swierczynski \& Wisniewski, 2008).
} 
definición de categorías compartidas por las tres asociaciones para reconocer los productos de investigación-creación. Dichas categorías comunes fueron llevadas por cada representante de asociación a sus bases para consulta y validación, con lo cual se presenta a Colciencias. Así, se trabajó conjuntamente en la construcción de tablas de requisitos de reconocimiento de "existencia" y de unos parámetros y niveles de "calidad". Luego, se hizo una traducción de niveles o grados de valoración congruente con el "algoritmo" del modelo de Colciencias para el cálculo, ponderación y comparación de la producción. Por último, se llevó la propuesta de modelo a la mesa y se negoció en su versión acabada ante Colciencias, organismo nacional encargado de fomentar la producción de conocimiento, construir capacidades para la ciencia, tecnología e innovación, y propiciar la circulación y usos de los mismos.

Iniciando por el trabajo con los referentes, se estudiaron los casos de Chile, Italia y Australia por ser modelos construidos desde el Estado y se desechó el modelo de México por estar basado en un sistema de empresas privadas de certificación. Se resaltó el sistema de la Universidad de Chile que establece criterios para la valoración de productos de creación basados en tres listas de espacios de exposición o eventos en los que se presentan las obras que por su trayectoria dan mayor o menor puntaje. Cada lista incluye sólo espacios del ámbito internacional, nacional o espacios que no necesariamente cuentan con mecanismos de selección (Universidad de Chile, 2013). Es importante aclarar que este sistema de valoración no funciona nacionalmente.

En Italia, desde 2013 el Ministerio de la Instrucción e Investigación Universitaria reconoce la profesión de 'docencia artística', reglamentada desde el sistema AFAM (instituciones de alta formación artística, musical y coréutica). Este sistema, adoptado por las academias de bellas artes, los conservatorios y la Academia Nacional de Danza, cuenta con un modelo propio de medición y escalafonamiento Ilamado 'Graduatoria para las AFAM' (Ley 143, 2004, 
Graduatoria Nacional). El modelo permite clasificar a los docentes contratados y aspirantes a puestos de trabajo, de acuerdo con un criterio propio que evalúa la calidad de la producción artística (obras, existencia, circulación, reconocimientos) y la experiencia académica (formación, experiencia, cargos, docencia, publicaciones e investigación) en foma separada y complementaria (AFAM - MIUR, 2017).

Finalmente, en el caso australiano The Australian Research Council reconoce los resultados de los procesos de creación como 'productos de investigación no tradicionales', evaluados con el apoyo de pares que siguen las directrices y criterios del sistema Excellence in Research for Australia (ERA). Los criterios de evaluación incluyen los antecedentes de la investigación dentro de su área de conocimiento y contexto, la contribución de la investigación en términos de innovación y nuevo conocimiento, y la importancia de la investigación estimada con base en la evidencia de su excelencia (Australian Research Council, 2015).

El caso chileno hace énfasis en la importancia de los espacios de circulación y apropiación social que dan relieve a la producción, pues se asume que debe respaldarse no solo en la trayectoria o antigüedad del espacio, sino en los mecanismos de selección por pares ciegos y jurados. Del caso italiano se acoge la particularidad del reconocimiento de la producción académica (títulos) y además de la producción artística (obras) avalada por el propio sistema del arte y la cultura pero nuevamente sustentada en premios, reconocimientos y procesos de curaduría, exigiéndose que esté claramente vinculada a procesos institucionales de investigación-creación. El caso de Australia arrojó luces sobre la necesidad de valorar la calidad más allá de lo académico por el impacto en las industrias creativas y culturales; no obstante quedan temas aún por resolver como la valoración de la producción identitaria y simbólica en contextos de alta diversidad cultural que transcurren fuera de la academia, 
como la valoración en términos de las lógicas de medición de audiencias, de difusión y circulación en la era digital (Domínguez, 2015).

\section{La investigación creación en la producción de nuevo conocimiento}

Partiendo de esas experiencias, los integrantes ahondaron en las raíces epistemológicas de esos modelos. Estas y otras referencias determinaron el ámbito de debate en la mesa AAD que derivó en el trabajo alrededor del concepto de investigación-creación (Bonilla et al., 2017) como un marco que da cuenta del resultado de una polinización cruzada entre disciplinas, que permite potencializar procesos y resultados propios de las artes, la arquitectura y el diseño, (AAD en adelante), además de dar una trazabilidad a la práctica creativa para evidenciar su rigurosidad en la generación de nuevo conocimiento (Colciencias, 2015) $)^{3}$.

Actualmente, dichos productos y procesos son reconocidos en el modelo de medición de Colciencias como actividades de generación de nuevo conocimiento, productos de nuevo conocimiento, desarrollos tecnológicos o innovaciones, aun si sus dinámicas y productos se diferencian de los de la investigación científica o la innovación tecnológica, que tradicionalmente hacen parte del SNCTI.

Por otro lado, estos debates también demostraron el impacto de la investigacióncreación en el desarrollo del país, teniendo en cuenta que los contenidos creativos y culturales que se producen, no solo generan experiencias estéticas sino también son transferibles al sector de las industrias creativas y culturales. Un ejemplo es el caso de la industria cinematográfica en donde a partir de

\footnotetext{
${ }^{3}$ Aunque existen conceptos similares como el de investigación artística (Hernández, 2006) o investigación basada en la práctica (Scrivener, 2002; Candy, 2006), se prefiere investigación-creación o 'creation-based research', pues enfatiza en el ejercicio creativo de producción de conocimiento común a las áreas de artes, arquitectura y diseño (Findeli et al., 2008).
} 
realidades imaginadas se ha promovido un desarrollo tecnológico e innovación que ha impactado diferentes áreas del conocimiento (Delgado, 2016).

Así, este artículo presenta el proceso de construcción del sistema de medición de los productos de investigación-creación, diseñado y puesto en práctica por la Mesa AAD, al igual que sus resultados iniciales obtenidos a través de la Convocatoria 737 de Colciencias para el Reconocimiento y Medición de Grupos de Investigación, Desarrollo Tecnológico o de Innovación y para el Reconocimiento de Investigadores de 2015.

\section{La investigación acción participativa y la mesa de negociación como una práctica de innovación social}

El proceso inicia en 2012, cuando Colciencias invita, en un evento en la Universidad Jorge Tadeo Lozano, a la comunidad académica para presentar el modelo de medición de investigadores y grupos de investigación en Colombia, modelo que habría de aplicar en su medición del 2013. A la reunión asisten representantes de varias universidades colombianas y se manifiesta el descontento por no sentir que la producción de los creadores de las artes el diseño y la arquitectura, estuviese incorporada. Como respuesta, meses después Colciencias invita a los representantes de las tres asociaciones de facultades de artes, arquitectura y diseño a sentarse y discutir la posibilidad de entrar a participar en una "mesa de negociación" (Dasanti, Hulsijn \& Van der Torre, 2000, p. 2) para desarrollar un sistema propio para medir su producción, siempre y cuando se integrara en el modelo y el algoritmo de Colciencias. Esto implicaba no solo aceptar y validar el modelo general, sino lograr un acuerdo para un modelo propio único para las tres asociaciones.

Así se delimitó la incertidumbre a partir de la cual se planteó la línea metodológica de investigación acción participativa de este proyecto, que 
principalmente tenía el propósito de generar una propuesta que más allá de ser encasillada en la producción científica de Colciencias, diera cabida a la producción AAD del país en el SNCTI.

Tal vez de forma intuitiva, fueron las prácticas colaborativas propias de los ecosistemas creativos las que ayudaron en ese proceso de negociación. Muchas de las formas del arte y el diseño colaborativo plantean la posibilidad de metabolizar el espíritu transformador de un determinado momento histórico dando lugar a procesos digestivos o subversivos. De Bruyne y Gielen (2011) trazan unos ejes que en dos extremos ponen estas categorías y en el otro sentido trazan un eje entre lo personal-relacional y lo colectivo-relacional. Si se toma la metáfora de lo coompetitivo ${ }^{4}$, se trataba de competir con el estamento y colaborar entre los proponentes (Osipov, 2013). Así se establecieron lugares comunes entre quienes competían como creadores-investigadores y se constituyó un ecosistema de trabajo participativo.

Los representantes de las universidades asimilaron la propuesta de Colciencias, pero se abogaron por un modelo que asumiese la diversidad dentro de la totalidad, por lo que el resultado estaba lejos de ser totalmente digestivo y asimilador, para ser potencialmente provocador y transformador, pero sin confrontación, promoviendo el cambio desde dentro y no tratando de modificar el sistema entero. Esta oportunidad y el reto de llegar a un lugar en el que nunca se había estado, motivó a la Mesa AAD a impulsar el desarrollo de un sistema particular para la medición de los productos y procesos de investigación-creación que complementa el modelo de medición usado por Colciencias en las convocatorias nacionales de medición de investigadores y grupos de investigación, pues antes de la intervención de la Mesa AAD, el modelo solo consideraba la generación de conocimiento a través de la investigación científica.

\footnotetext{
${ }^{4}$ Nuevo término desarrollado para la gerencia estratégica de cooperación y competencia simultánea donde: compito con los otros creadoresproductores y cooperó con los del propio ecosistema creativo. Pero mayormente no sucede de forma permanente, sino en alianzas por proyectos (Osipov, 2013)
} 
Con base en el concepto de investigación-creación la mesa AAD construye un primer prototipo de un modelo de medición, descrito en el documento conceptual de la Convocatoria nacional 693 para el reconocimiento y medición de grupos de investigación, desarrollo tecnológico e innovación de 2014, capítulo 2.1.3.5 Otros productos resultado de la creación o investigacióncreación en arte, arquitectura y diseño, y además se incluye el formulario Productos de arte, arquitectura y diseño (productos AAD) en la plataforma en línea de medición de grupos de investigación CVLAC5.

Con los resultados positivos obtenidos de esa convocatoria se crea una línea base que evidencia la necesidad que tienen los investigadores-creadores del país de contar con un sistema que valore sus productos de acuerdo con la producción que caracteriza estas áreas; al impacto que generan en sus disciplinas y en el desarrollo del país; y que se inserte dentro de la lógica de clasificación establecida por el sistema de medición nacional.

Así, el proceso de formulación y negociación situado en el marco de este experimento de la mesa de trabajo / mesa de negociación, se desarrolló bajo una dialéctica entre los modelos de investigación experimental e incremental, buscando otros planos de contexto, donde la libertad y la significación de las acciones cotidianas de la realidad humana pudieran tener un espacio con mecanismos de investigación cualitativa (Miles y Huberman, cit. por Pérez, 2001a; (Quintana \& Montgomery, 2006) Por lo tanto, modelos como la investigación acción participativa - IAP, donde se plantean las respuestas a partir de un examen de la situación de las comunidades desde la práctica acopiada del investigador, facilitaron la integración activa en el proceso de los miembros de las comunidades académicas en calidad de investigadores y a su vez actores de la investigación (Castillo, 2004, p. 16). Así, la gestión

${ }^{5}$ Es el formato que permite a los investigadores adscritos a un grupo de investigación reconocido y valorado por Colciencias, publicar sus curriculum vitae et studiorum, con los respectivos productos y aportes al conocimiento. 
global del conocimiento parece asemejarse a este tipo de hibridación del "co-laboratorio" entendido como la capacidad de contratación o negociación (comprometer), aliado a la de formar el liderazgo responsable (educar) y por último a la organización para servir objetivos comunes (habilitar)" (Muff 2014, p. 19). Lo mismo puede decirse del valor de esta experiencia y del riesgo que significó, pues todo había podido salir mal, porque se trataba de hacer algo que nunca se había hecho antes, pues, se pregona el innovar y poco se entiende de lo difícil que es aprender a hacer lo que nunca se ha hecho, ser como nunca se ha sido y atreverse a perder una identidad por abrazar lo desconocido (Fernández \& Johnson, 2015).

Este proceso solo podía ser legitimado regresando a la base y consultando dentro de cada asociación para regresar legitimados. Y así fue como se logró no solo un diálogo entre los investigadores sino también trabajar para construir el nuevo modelo. Así, se estaba en lo que se llama desde la teoría " $U$ " construyendo nuevas identidades, nuevos "conceptos de comunidad" (Mirvis, en Muff Ed. 2014, p. 175).

Finalmente, conviene aclarar que la investigación que este artículo presenta no fue fruto de un proceso de investigación ceñida únicamente a criterios académicos o científicos. Fundamentalmente se trató de un proceso político de investigación y negociación, realizado entre dos partes en una "mesa" de negociación: por una parte las universidades representadas en las asociaciones y por la otra el Estado - Colciencias. Este proceso de investigación no terminó en un informe, ni un artículo, ni un producto o una patente, sino en un modelo avalado y aplicado por Colciencias con un carácter de "norma" o protocolo con validez legal para medir, valorar y hacer una gradación de la producción de nuevo conocimiento en las áreas de AAD. 


\section{Formulación de conceptos y protocolos para la construcción del sistema de medición de los productos resultado de investigación-creación}

Para el reconocimiento y medición de grupos de investigación e investigadores del SNCTI del país, Colciencias utiliza el sistema de información ScienTI que incluye los módulos CvLAC, en donde los investigadores registran en línea sus hojas de vida y producción intelectual, y GrupLAC, donde se registran los grupos de investigación a los que los investigadores vinculan sus productos. De esa forma se leen relaciones y cualidades de producción (i.e., la cantidad y carácter de los productos en el tiempo, circulación y difusión), la cohesión del grupo a través de la coautoría de miembros de un mismo grupo, y la cooperación a través de la coautoría entre miembros de diversos grupos.

Además, de medir periódicamente la producción de los grupos e investigadores, ScienTI permite leer dinámicas de desarrollo en temas y campos del conocimiento. Su base de datos aplica un modelo matemático para calcular y valorar ponderadamente la producción registrada en sus bases de datos. Estos resultados permiten categorizar los grupos de investigación y clasificar a los investigadores con el fin de determinar la calidad de su labor y trayectoria, lo que influye en la distribución de recursos en las convocatorias de Colciencias para financiar proyectos de investigación.

Igualmente, los resultados de esta medición son tenidos en cuenta en los procesos de acreditación de programas académicos y en el ranking MIDE, que utiliza el Ministerio de Educación Nacional para la clasificación de las instituciones de educación superior. 
Aproximación a los criterios para establecer el modelo de medición para productos resultado de la investigación-creación

Es evidente que el debate y el conflicto interno en las universidades desatado por la búsqueda del reconocimiento de la investigación artística, iniciado por Borgdorff constituye un tema político y epistemológico que supera un simple problema de fronteras de conocimiento e identidad académica y rompe equilibrios de poder jerárquico con implicaciones en decisiones de prioridad de inversión y presupuesto en áreas como la ciencia y la tecnología basados históricamente en el imperio de la publicación científica validando ediciones como el (Borgdorff, 2012). Ese mismo debate sobre la producción artística y la investigación desde el realismo constructivista que se deslinda del paradigma empírico-deductivo de las ciencias exactas o del empírico-descriptivo de las ciencias sociales ha sido ampliado enormemente en los últimos años caminando hacia una perspectiva performativa del conocimiento (Borgdorff, 2012), estando muy lejos de haberse consolidado completamente. Patricia Leavy (1975, reeditado en 2015) inicia un trabajo valioso sobre las prácticas de investigación basadas en las artes, desde el aporte a la investigación social a partir de la escritura creativa, la indagación narrativa, la ficción o la poesía como un paradigma alternativo que tiende puentes entre arte y ciencia. Biggs y Karlsson (2012) realizan una importante compilación que nuevamente aborda los temas del poder de la política universitaria de la investigación basada en la experiencia, ahonda en los temas de la pluralidad del arte como otra forma de investigación que recoge defensores y detractores para plantear el tema de la medición de la producción de conocimiento artístico y el rol del artefacto en la investigación basada en las artes; el rol de las comunidades, los valores, las convenciones y las acciones propias de la cognición artística y creativa cuya producción objetual y visual, la escritura creativa, la música, es conocimiento encarnado, performativo, interactivo, fenomenológico que permite la heterogeneidad, la intuición y el discernimiento con rigor en 
prácticas transformadoras culturales factibles de ser valoradas cualitativamente como nuevo conocimiento.

Para delimitar los productos que han de ser valorados, la construcción del modelo de medición exige establecer un marco de requerimientos sobre el que se construyen los parámetros requeridos por el modelo vigente. Como punto de partida, la mesa nacional AAD construyó la siguiente definición:

Se entiende por Obras, Diseños y Procesos de Nuevo Conocimiento, provenientes de la Creación en Artes, Arquitectura y Diseño, aquellas obras, diseños o productos resultantes de los procesos de creación que implican aportes nuevos, originales e inéditos al arte, a la arquitectura, al diseño, a la cultura y al conocimiento en general a través de lenguajes simbólicos que expresan, interpretan y enriquecen de manera sustancial la vida intelectual, emocional, cultural y social de las comunidades humanas. Estos productos provienen de proyectos de investigación, creación o investigación-creación, debidamente aprobados mediante convocatorias internas o externas o avalados por organizaciones de reconocido prestigio institucional de carácter local, regional, nacional e internacional. (Colciencias, 2015, p. 38)

La construcción de esta definición sentó las bases para comprender la naturaleza de estos productos e iniciar un proceso de análisis cualitativo y determinar los parámetros de reconocimiento y valoración. Sin embargo, la gran diversidad de productos que abarca esta amplia definición presenta el problema de que una valoración generalizada perjudica ciertas tipologías, por lo que se buscó entender las características comunes que determinan la calidad de la gran diversidad de obras existentes, más allá de las disciplinas, y así formular un agrupamiento más apropiado. La idea de realizar una agrupación motivó a las asociaciones a convocar por medio de los decanos a los investigadores-creadores quienes con el apoyo de los representantes de las asociaciones ayudaron a construir unos listados o tablas de productos de sus respectivos campos; estableciendo así, un proceso participativo entre facultades y programas de AAD del país. 
No obstante, lejos de allanar el camino y facilitar el trabajo de la mesa, estos listados hicieron más evidente la dificultad y la inoperancia de un modelo basado en categorías provenientes de cada campo (técnicas, lenguajes o productos particulares). Más que productos de nuevo conocimiento sólo reflejaban productos finales del ejercicio profesional, sin ningún indicio sobre cómo dar cuenta de los valores y calidades de ellos o de los mecanismos útiles para reconocer sus aportes al conocimiento, desarrollo tecnológico e innovación del país. En el caso de ACFA, los miembros de facultades de arquitectura construyeron un listado con más de 60 categorías de productos y alrededor de 400 subcategorías $^{6}$. Ese abordaje se mostraba inapropiado y anacrónico, era evidente que las técnicas y lenguajes, los formatos y maneras de circular se habían disparado en la era digital, no era posible comparar y validar un listado tan grande y dinámico en las técnicas y las hibridaciones.

Los listados fueron llevados nuevamente a las respectivas asociaciones para que las categorías de clasificación de productos fueran discutidas de acuerdo con la realidad de cada campo disciplinar. Así, se acogieron las observaciones de los miembros de cada asociación quienes evidenciaron la inoperancia del criterio de la taxonomía por familias de productos, concluyendo la necesidad de identificar la convergencia, lejos de límites disciplinares y con mirada a los múltiples traslapes y relaciones existentes entre los tres campos ${ }^{7}$.

De esta manera, instigados por la idea de una convergencia despojada de identidades estocásticas de competencias o habilidades, de formatos y oficios se estableció una agrupación de acuerdo con la naturaleza temporal, resaltando el vínculo entre la acción de creación, la puesta en escena de la obra, y la estrategia de circulación de los productos/obras resultado de investigación-

\footnotetext{
${ }^{6}$ Taller 1: Construcción participativa de listado de categorías Bogotá; y Taller 2: Cotejo de las tres categorías según naturaleza temporal del producto, ambos realizados en Bogotá, ACFA, 2014.

${ }^{7}$ Criterios de evaluación de obras, diseños y procesos de nuevo conocimiento, provenientes de la creación en artes, arquitectura y diseño (documento de trabajo, ACOFARTES, ACFA, RAD, 10-26/07/2014).
} 
creación. Esto abre un punto de convergencia entre los tres campos, y evidencia cómo la práctica creativa propia de estas áreas no solo genera una gran variedad de productos sino también establece puntos comunes que trascienden los límites disciplinares. A continuación, la agrupación propuesta a partir de la naturaleza temporal de los productos de creación:

- Obra o creación efímera ${ }^{8}$ : Son las obras, diseños o productos, materiales e inmateriales, cuya existencia es de una duración limitada en el tiempo y el espacio, y cuya evidencia depende, por lo tanto, de la memoria reconstructiva. Son sus huellas, rastros, o registros los que corroboran su existencia y las hacen reconocibles. El registro debe ser repetible, exportable y verificable (EcuRed, 2017).

- Obra o creación permanente: Son obras, diseños o productos, materiales e inmateriales, cuya existencia pretende ser ilimitada en el tiempo. La presencia y persistencia del objeto que registra la obra o producto demuestra su existencia. Sin embargo, la obra o producto mismo predomina sobre el valor del registro.

- Obra o creación procesual ${ }^{9}:$ Son aquellas obras, diseños o productos materiales o inmateriales, en cuya naturaleza predomina la dinámica transformadora, sistémica y relacional; por esta razón tienen un carácter abierto y no están sujetas a un marco espacio temporal predeterminado. Generan impacto verificable pero no previsible material e inmaterial. El reconocimiento de este tipo de producto se basa en la existencia de indicadores cualitativos o cuantitativos que den cuenta de las dinámicas del proceso.

\footnotetext{
${ }^{8}$ Arte efímero. Es toda expresión artística concebida bajo un concepto de fugacidad en el tiempo, de no permanencia como objeto artístico material y conservable.

${ }^{9}$ Para mayor información, ver la definición que da Germano Celant del arte procesual como un dar cuenta del quehacer pragmático del arte a través de una identidad entre pensamiento y acción (citado en Argán, 1992, p. 77).
} 
A partir de esta agrupación, la Mesa AAD consideró pertinente realizar un mapeo nacional de estos productos, que le permitiera analizar las tipologías existentes y encontrar puntos comunes de calidad para determinar parámetros en términos de generación de las categorías de Colciencias: nuevo conocimiento, desarrollo tecnológico e innovación.

Con el apoyo de Colciencias, se recolectó a través de un formulario para productos de arte, arquitectura y diseño publicado en CvLAC (Convocatoria 693 de 2014) la información necesaria para realizar un sondeo de las obras o creaciones permanentes, efímeras y procesuales realizadas por investigadorescreadores a nivel nacional. Con el repositorio de información obtenido, la mesa AAD estableció los parámetros básicos de comparación y ponderación, para realizar la primera valoración de los productos propios de las artes, la arquitectura y el diseño en 2015. Este escenario planteaba no solo la necesidad de realizar una reflexión de carácter conceptual acerca del valor y la calidad, sino también el problema efectivo de la valoración subjetiva de carácter estético. De ahí, se proyectó la necesidad de estructurar un sistema objetivo afín al modelo del sistema de información ScienTI, sin poner en riesgo las formas propias de valoración del mundo artístico-académico de las artes, la arquitectura y el diseño.

La valoración de los procesos y los productos de creación en el desarrollo de una propuesta de medición de grupos e investigadores de artes, arquitectura y diseño

Es importante evidenciar que al incluir este tipo de procesos y productos resultado de investigación-creación en el SNCTI, se hace necesario el reconocimiento de diversas instancias de validación y difusión del conocimiento, propias de las artes, la arquitectura y el diseño como concursos, exposiciones, bienales entre otras. En estas instancias se realizan juicios sobre la calidad de las obras a partir de una 
evaluación por pares expertos (jurados, curadores, comisiones de expertos), que, aunque no corresponden a las formas tradicionales de evaluación de la actividad científica, por ejemplo la evaluación doble ciega; sí forman parte de la tradición de las disciplinas creativas, y refleja todo un sistema de evaluación, transferencia y apropiación (consumo cultural, formación de públicos, trayectoria) que es ampliamente aceptado dentro de las diferentes comunidades de creadores. Es evidente que estas instancias de valoración no se rigen por el formato de publicaciones académicas construido por el mundo científico ${ }^{10}$ y ponen en evidencia un sistema del arte distinto en sus formas de producción, validación y difusión de conocimiento completo (Leavy, 2015) y con una trayectoria importante, demostrando la existencia de otros sistemas de generación de conocimiento como mencionan Nowotny, Scott y Gibbons (2003). A pesar de ser estrategias muy distintas a las que se manejan en el ámbito científico, la rigurosidad de sus mecanismos de evaluación ha determinado parámetros propios que han establecido los referentes de calidad en la producción creativa de estas comunidades internacionalmente (Casas, 2013).

De acuerdo con la tipología y temática (cine, mobiliario, teatro, arquitectura, música, etc.), cada instancia y espacio de investigación-creación organiza comités de pares expertos, que estructuran los protocolos de evaluación que rigen sus evaluaciones para determinar la calidad de las obras o creaciones, que se sometan a estas instancias de valoración. En el caso de los museos y las galerías, para cada una de sus exposiciones se realizan procesos curatoriales estructurados sobre un proyecto expositivo que evalúa distintos aspectos para seleccionar las piezas artísticas que harán parte de sus colecciones y exposiciones (Betancourt, 2012). Mecanismos similares se manejan en concursos, bienales y festivales, entre otros escenarios, promoviendo nuevas formas de valoración de conocimiento que se interconectan con su transferencia y apropiación social, que en la mayoría de casos son también consideradas 
instituciones mediadoras entre contenidos creativos/culturales y la sociedad (Betancourt, 2012; Hernández, 2014).

Premios, menciones, selección para exposiciones permanentes o temporales, etc., son los reconocimientos que estas instancias otorgan y que hacen de una obra o creación un referente de calidad en las mencionadas disciplinas. Por ejemplo, es difícil cuestionar el valor artístico de una obra cinematográfica que haya sido reconocida por el Festival de Cannes, el cual tiene una trayectoria y experiencia en el tema de más de 70 años, o de un producto premiado en el concurso de diseño Compasso d'Oro, que con sus 50 años de funcionamiento ha realizado 24 ediciones.

Este reconocimiento de las formas vigentes de legitimación y valoración de los campos creativos permitió a la mesa AAD proponer la articulación de estas instancias de evaluación con el modelo vigente de medición de Colciencias, teniendo en cuenta que estas instancias de valoración tienen su propia trayectoria, experiencia y credibilidad. Esto se hizo de manera análoga al reconocimiento que hace este modelo del sistema de revistas científicas indexadas; que, aunque se encuentra mucho más estandarizado y cuenta con procesos aceptados ampliamente en el mundo académico, también contiene un alto nivel de subjetividad. Por esta razón, fue necesario establecer que los procesos y productos resultado de investigación-creación que quisieran hacer parte activa del SNCTI deberían someterse a la criba cruzada de revisiones críticas, auto-reflexivas y de expertos, que logren determinar la rigurosidad del proceso creativo y el impacto de sus resultados de nuevo conocimiento, como lo recomienda el modelo británico de evaluación de la investigación (The Research Assessment Exercise -RAE- citado en Hernández, 2006). 
Al articular la valoración de una obra con el impacto de la instancia que la evalúa fue posible estructurar los siguientes criterios de calidad para la valoración de los productos de nuevo conocimiento resultado de investigación-creación:

- Tipo de reconocimiento: de acuerdo con el tipo de reconocimiento que reciba una obra o creación en una instancia de valoración (primer puesto, nominación, mención especial, expositor invitado, selección para muestra, etc.) se establece uno de los parámetros de calidad de la obra.

- Trayectoria de la instancia que evalúa: la trayectoria de una instancia de evaluación da fe de su propia experiencia, credibilidad e impacto. Estos aspectos permiten respaldar la calidad de las obras o creaciones evaluadas. Debido a la naturaleza automática del modelo de medición, se determina esta trayectoria con la edad de la instancia de validación.

- Mecanismo de selección: dependiendo de la existencia o no de un mecanismo de selección (jurado internacional, revisión anónima, invitación especial, curaduría, etc.) y los protocolos de evaluación que se manejen, se evidencia un juicio de pares ciegos que valida y fortalece el reconocimiento de calidad e impacto de una obra. Toda instancia de valoración debe contar con un mecanismo visible de selección o curaduría

- Ámbito (local, nacional e internacional): el ámbito es uno de los parámetros que permite determinar el impacto y visibilidad de una obra o creación. Para efectos de este modelo, se define teniendo en cuenta, no el lugar de presentación de la obra o producto, sino la procedencia de la mayoría de participantes en el espacio o evento correspondiente a la instancia de valoración. 
Estos criterios permiten definir cuatro categorías de productos de nuevo conocimiento, resultado de la creación:

- $\quad$ AAD A1: Obras o productos que tienen premio o distinción en eventos o espacios del ámbito internacional, con trayectoria superior a 10 años.

- AAD A: Obras o productos que han sido premiados o distinguidos en eventos o espacios del ámbito nacional, con trayectoria superior a 10 años. En esta categoría también están los que han sido seleccionados para su presentación en espacios o eventos del ámbito internacional y trayectoria superior a 10 años.

- AAD B: Obras o productos que han obtenido premio o distinción en eventos o espacios del ámbito local y trayectoria superior a 10 años. En esta categoría también se encuentran los que han sido seleccionados para su presentación en espacios o eventos del ámbito nacional y trayectoria superior a 10 años.

- AAD C: Obras o productos que han sido seleccionados para su presentación pública en eventos o espacios con mecanismo visible de selección o curaduría, con impacto local.

Como se mencionó, todo evento o espacio de valoración debe contar con un mecanismo visible de selección o curaduría.

Articulación con el modelo matemático, para una inclusión integral de la investigación-creación en los procesos de medición de Colciencias

Otro tema delicado en la negociación por su menor maleabilidad y posibilidad de ser modificado era el modelo matemático de distribución de pesos entre los diferentes productos que ingresan en el algoritmo de medición del modelo de Colciencias. Se sabe que cuando los valores de varios productos son ponderados y a cada uno es atribuido un peso relativo, la suma hace que unos 
adquieran una mayor importancia que otros, logrando mover la balanza en un sentido o en otro.

Existe entonces una relación entre el modelo de medición de Colciencias y el "nuevo" modelo de la mesa AAD que plantea un proceso incremental de cambio en las prácticas entre las facultades de artes, arquitectura y diseño (Gómez \& Martínez, 2014). Esto en relación con las otras facultades con mayor peso específico en la sociedad del conocimiento y al mismo tiempo entre estas facultades de un ámbito creativo con un ente del estado colombiano que regula y aplica los modelos de medición de productividad científica y tecnológica. Era evidente que el valor de los productos de AAD reconocidos por el modelo debería sufrir una pérdida de valor o peso respecto de aquellos vinculados a la investigación científica o el desarrollo tecnológico. Pues bajo las reglas tácitas de la mesa de negociación, no era visible para la ciencia tradicional el cómo equilibrar esas valoraciones haciéndolas más equitativas, pero se entendió, que este proceso de nuevo equilibrio hace parte del debate sobre el poder académico de ciertos sectores académicos universitarios que este primer "nuevo" modelo está aún lejos de poder modificar. Muy seguramente, como en otras naciones, esto no lo resolverá jamás una reflexión o un debate realizado en la sociedad del conocimiento, es el peso económico de sectores como el de las llamadas industrias creativas y culturales en los factores de desarrollo y crecimiento económico los que harán inclinarse la balanza y variar ese algoritmo que decide en buena medida a dónde se dirigen los fondos estatales que hoy se encaminan a la ciencia, la tecnología y la innovación $(\mathrm{Ci}+\mathrm{T}+\mathrm{I})$, y a futuro en la creación $(\mathrm{Ci}+\mathrm{T}+\mathrm{I}+\mathrm{Cr})$.

Por este motivo, planteó la necesidad de entender a profundidad el modelo matemático usado por Colciencias para asignar puntajes a los productos de nuevo conocimiento, tecnología o innovación, para que de esa forma los productos de la categoría obras o productos de investigación-creación en artes, 
arquitectura y diseño pudieran ser ponderados y homologados en igualdad de condiciones con los productos científicos tradicionales. En el modelo matemático, todas las categorías de productos tienen un ponderador relativo entre 1 y 10. Los productos de mayor calidad de esa categoría reciben 10 puntos de peso relativo (Tabla 1).

Tabla 1. Categorías de productos de nuevo conocimiento, resultado de la investigación-creación en Artes, Arquitectura y Diseño. Fuente: Modelo conceptual para la convocatoria 737 (Colciencias, 2015).

\begin{tabular}{|c|c|c|}
\hline Categoría & Requerimientos de calidad & $\begin{array}{c}\text { Peso } \\
\text { relativo }\end{array}$ \\
\hline AAD A1 & $\begin{array}{l}\text { La obra o producto ha obtenido premio o distinción } \\
\text { en eventos o espacios del ámbito internacional, con } \\
\text { mecanismo visible de selección o curaduría y trayectoria } \\
\text { superior a } 10 \text { años. }\end{array}$ & 10 \\
\hline AAD A & $\begin{array}{l}\text { La obra o producto ha obtenido premio o distinción en } \\
\text { eventos o espacios del ámbito nacional, con mecanismo } \\
\text { visible de selección o curaduría y trayectoria superior a } \\
10 \text { años. } \\
\text { o } \\
\text { La obra o producto ha sido seleccionada para su } \\
\text { presentación en espacios o eventos del ámbito } \\
\text { internacional con mecanismo visible de selección o } \\
\text { curaduría y trayectoria superior a } 10 \text { años. }\end{array}$ & 8 \\
\hline AAD B & $\begin{array}{l}\text { La obra o producto ha obtenido premio o distinción en } \\
\text { eventos o espacios del ámbito local con mecanismo } \\
\text { visible de selección o curaduría y trayectoria superior a } \\
10 \text { años. } \\
\text { o } \\
\text { La obra o producto ha sido seleccionada para su } \\
\text { presentación en espacios o eventos del ámbito nacional } \\
\text { con mecanismo visible de selección o curaduría y } \\
\text { trayectoria superior a } 10 \text { años. }\end{array}$ & 6 \\
\hline AAD C & $\begin{array}{l}\text { La obra o producto ha sido seleccionada para su } \\
\text { presentación pública en eventos o espacios con } \\
\text { mecanismo visible de selección o curaduría, con } \\
\text { impacto local. }\end{array}$ & 4 \\
\hline
\end{tabular}


Los pesos relativos son la base para clasificar los productos, resultado de procesos de investigación, desarrollo tecnológico o de innovación de acuerdo con su aporte al estado del arte, de la técnica en su respectiva área del saber. Aquellos que han hecho aportes más significativos se clasifican en el grupo Top, seguidos de los $A$ y $B$. Con base en esta clasificación, complementada con otros indicadores que caracterizan cada grupo de investigación en seis perfiles, el modelo de Colciencias (2015) define los puntajes que procuran la clasificación ordinal de los grupos (A1, A, B, C y D) y la clasificación de sus investigadores (sénior, asociado y junior ${ }^{11}$ ).

La ventana de observación de los productos de creación es de diez años teniendo en cuenta que tienen una vida y unas formas de apropiación que difieren de las de los productos científicos. Una obra puede obtener varios reconocimientos en forma gradual a través del tiempo y por ello se decidió que la ventana de observación se activará, no con la fecha de creación de la obra, sino con la fecha de su presentación en la instancia de validación. De esta forma, un producto de creación que después de varios años de creado empieza a ser reconocido y divulgado, puede tener una oportunidad de valoración.

De esta manera. se hizo posible incorporar de forma integral, en la Convocatoria 737 del 2015, los productos AAD al modelo conceptual de medición de Colciencias. Esta vez con los insumos particulares requeridos por el modelo matemático, con el fin de abrir por primera vez las puertas a la valoración y puntuación de productos propios de las áreas de artes, arquitectura y diseño en Colombia.

\footnotetext{
${ }^{11}$ Detalles del modelo matemático utilizado por Colciencias puede ser consultado en el Capítulo III. del documento Modelo de medición de grupos de investigación, desarrollo tecnológico o de innovación y de reconocimiento de investigadores del sistema nacional de ciencia, tecnología e innovación, 2015, pp. 65-79.
} 


\section{Otros productos incluidos en el sistema de medición resultado de investigación-creación}

Además de los criterios para la valoración de los productos de nuevo conocimiento resultado de investigación-creación, la mesa AAD propuso la inclusión de otros productos homologables a otros ya existentes dentro del modelo.

Dentro de la tipología de productos resultado de actividades de apropiación social de conocimiento, que deben incluir la formación de públicos, se propusieron los siguientes:

- La organización o participación en eventos artísticos y culturales es homologable a la participación en eventos científicos.

- Participación en talleres de creación, residencias artísticas o laboratorios. En estos casos la valoración tiene en cuenta aspectos similares a los de los resultados de creación: se espera que el participante acceda al simposio, taller, residencia, o laboratorio ${ }^{12}$ a través de una selección o curaduría y que la actividad sea organizada por una entidad con trayectoria en el campo y que cumpla con un registro del proceso creativo y un protocolo de validación con pares externos.

Dentro de la tipología de productos resultado de actividades de desarrollo tecnológico e innovación, la mesa AAD propuso los siguientes productos:

- Acuerdos de licencia para la explotación de obras protegidas por derechos de autor. Este producto se puede derivar de los resultados de creación que cumplan con los requisitos para ser considerados como nuevo conocimiento.

\footnotetext{
${ }^{12}$ Se incluyen workshops, clínicas, coros, ensambles, bandas y los simposios artísticos de escultura que dieron origen a esta modalidad de espacios de creación-formación e intercambio y convivencia donde no se exhibe, sino que durante varios días se trabaja, se produce y circula la creación, predominando el valor experiencial tipo laboratorio.
} 
- Empresas creativas y culturales. Es decir, las spin-off que se generan como resultado de actividades registradas de creación o investigacióncreación.

- Consultorías en procesos de investigación-creación en arte, arquitectura y diseño.

Con estos tres productos, más allá de reconocer prácticas existentes, se fomenta una articulación cada vez mayor entre la creación realizada en el ámbito académico y las industrias culturales y creativas, en correspondencia con el propósito del sistema de ciencia, tecnología e innovación, consistente de fomentar la generación de conocimiento nuevo que impacte el sistema productivo (Colciencias, 2015).

\section{Resultados}

El sistema diseñado por la Mesa AAD (autores del presente artículo), para la medición de los productos resultado de investigación-creación, fue implementado en la convocatoria 737 de 2015 de Colciencias. De acuerdo con los resultados de la convocatoria, el registro de los productos de AAD presentó un aumento del 766\% respecto a la Convocatoria 693 de 2014. Del total de los productos registrados en CvLac para la convocatoria 737, el $32 \%$ de los productos corresponde a resultados de actividades de generación de conocimiento, el 30\% a actividades relacionadas con la formación de recurso humano, el $28 \%$ a resultados de actividades de apropiación social del conocimiento, y el $10 \%$ a resultados de actividades de desarrollo tecnológico e innovación. Adicionalmente, de la convocatoria 737 a la convocatoria 781 de 2017 el incremento de la producción aumentó un 670\% (Tabla 2). 
Tabla 2. Resultados de la medición de productos de artes, arquitectura y diseño de la convocatoria 737 de 2015 y 781 de 2017. Fuente: Participación y aportes de la comunidad de artes, arquitectura y diseño en el Modelo de Reconocimiento y Medición de Grupos de Investigación Desarrollo Tecnológico o de Innovación y en el Reconocimiento de Investigadores del Sistema Nacional de Ciencia, Tecnología e Innovación (Colciencias, Dirección de Fomento a la Investigación, 2016).

\begin{tabular}{llcc}
\hline Tipología general de producto & Tipo específico de producto & $\begin{array}{c}\text { Convocatoria } \\
\mathbf{7 3 7}\end{array}$ & $\begin{array}{c}\text { Convocatoria } \\
\mathbf{7 8 1}\end{array}$ \\
\hline $\begin{array}{l}\text { Productos resultados de } \\
\begin{array}{l}\text { actividades de generación de } \\
\text { nuevo conocimiento }\end{array}\end{array}$ & $\begin{array}{l}\text { Obras o productos de } \\
\text { investigación-creación en artes, } \\
\text { arquitectura y diseño }\end{array}$ & 987 & 2839 \\
& &
\end{tabular}

\begin{tabular}{llll}
\hline $\begin{array}{l}\text { Productos resultados de } \\
\text { actividades de desarrollo } \\
\text { tecnológico e innovación }\end{array}$ & Empresas creativas y culturales & 7 & 40 \\
\cline { 2 - 3 } & $\begin{array}{l}\text { Consultoría científico } \\
\text { tecnológica e informe } \\
\text { técnico - consultoría en artes, } \\
\text { arquitectura y diseño }\end{array}$ & 187 & 464 \\
\hline & $\begin{array}{l}\text { Acuerdos de licencia para la } \\
\text { explotación de obras protegidas } \\
\text { por derecho de autor }\end{array}$ & 0 & 813 \\
&
\end{tabular}

\begin{tabular}{llll}
\hline \begin{tabular}{l} 
Productos resultados de $\begin{array}{l}\text { actividades de apropiación } \\
\text { social del conocimiento }\end{array}$ \\
\cline { 2 - 3 }
\end{tabular} & Talleres de creación & 0 & 244 \\
\hline $\begin{array}{l}\text { Productos de actividades } \\
\begin{array}{l}\text { relacionadas con la formaciónticos } \\
\text { de recurso humano }\end{array}\end{array}$ & $\begin{array}{l}\text { Proyecto de investigación y } \\
\text { creación }\end{array}$ & 1840 \\
\hline
\end{tabular}

\begin{tabular}{lll}
\hline Total de productos & 1359 & 6735 \\
\hline
\end{tabular}


Estos resultados reflejan por un lado el interés de las comunidades académicas de artes, arquitectura y diseño del país por participar con estos nuevos productos en las convocatorias de medición de investigadores y grupos de investigación. De otro lado, dan cuenta de la gran cantidad de productos resultado de procesos de creación que necesitaban un espacio claro dentro del modelo de medición de Colciencias. Y finalmente, también evidencian la necesidad de generar consciencia sobre cómo, al reconocer la creación como proceso de generación de conocimiento, se crea una mayor expectativa por la calidad y potencial de impacto de los productos realizados en ambientes académicos.

\section{Conclusiones}

La inclusión de estos productos en el modelo de medición trasciende el simple reconocimiento de los resultados de creación. Esta iniciativa proyecta una articulación con los mecanismos de acceso a recursos para investigación, y los escalafones docentes para bonificación y ascenso. Pero sobre todo evidencia cómo los procesos de creación, propios de estas disciplinas, realizan aportes determinantes al nuevo conocimiento, al desarrollo tecnológico y a la innovación; aspectos fundamentales en el desarrollo social, cultural y económico del país.

Este esfuerzo busca impulsar un sistema que fomente la creación artística, arquitectónica y de diseño de alto nivel en los grupos de investigación, y facilitar su incorporación al sector productivo, de manera que las condiciones de la creación en la academia puedan efectivamente traducirse en contenidos originales que disparen la competitividad de las industrias creativas y culturales en Colombia. 
Como emergió del proceso de socialización y aplicación del modelo, quedan asuntos pendientes como la incorporación de sistemas de medición que permitan incorporar el impacto que tienen las obras, productos o procesos de investigación-creación en el desarrollo y el comportamiento de las industrias creativas y culturales, así como la valoración de calidad que incorpore el impacto en la formación de públicos y audiencias por apropiación y circulación.

Los resultados demostraron cómo es posible generar una mayor equidad en el reconocimiento de productos de arte, arquitectura y diseño respecto de los productos científicos presentados en el modelo existente.

Por lo anterior, es importante entender el trabajo de la Mesa nacional AAD como un proceso abierto y permanente que requerirá del esfuerzo de sus sostenedores actuales y otros nuevos, para mantener vivo y en desarrollo dicho escenario, teniendo en cuenta que esta es apenas una etapa que se cumple en el proceso focalizado a la construcción del Programa Nacional de las Artes, la Arquitectura y el Diseño (como entidad que fomente los procesos de creación para la generación del nuevo conocimiento, desarrollo tecnológico, innovación y competitividad del país), que se propuso como objetivo fundamental desde la creación de la mesa en el 2013.

\section{Referencias}

Argán, G.C. (1992). El arte moderno. Madrid: Akal.

Asprilla L. I. (2013). El proyecto de investigación-creación: la investigación desde las artes. Santiago de Cali: Instituto Departamental de Bellas Artes.

Australian Research Council. (2015). ERA 2015. Excellence in Research for Australia. Submission guidelines. ISBN 978-0-9924254-8-7 (print), ISBN 978-0-9924254-9-4 (digital), ISBN 978-0-9943687-0-6 (HTML) 
Betancourt, C. (2012). Conceptos generales de museología. En: Ministerio de Cultura (Ed.), Museología, curaduría, gestión y museografía. Manual de producción y montaje para las Artes Visuales. ISBN: 978-958-753-044-5

Recuperado de http://www.museoscolombianos.gov.co/fortalecimiento/ comunicaciones/publicaciones/Documents/manual_artes_visuales_ mincultura.pdf

Biggs, M. A. R., \& Karlsson, H. (2011). Evaluating quality in artistic research. In M. A. R. Biggs \& H. Karlsson (Eds.), The Routledge companion to research in the arts (pp. 405-424). London: Routledge. ISBN-10: 0415581699

Bonilla, H., Cabanzo, F., Delgado, T., Hernández, O., Niño, A. y Salamanca, J. (2017) Apuntes sobre el debate académico en Colombia en el proceso de reconocimiento gubernamental de la creación como práctica de generación de nuevo conocimiento, desarrollo tecnológico e innovación. Cuadernos de Música, Artes Visuales y Artes Escénicas, 12 (2), 281-294. DOI https://doi.org/10.11144/javeriana.mavae13-1.asda

Borgdorf, H. (ed) (2012). The conflict of the faculties. Perspectives on artistic research and academia. Amsterdam: Leiden University Press. ISBN 9789087281670. http://hdl.handle.net/1887/18704

Candy, L. (2006). Practice based research: A guide. En: CCS Report, 2006---v1.0 1, 1-19. Sidney: University of Technology. http://www. creativityandcognition.com/

Casas, M.V. (2013). Una aproximación al estado del arte en Colombia y otros países sobre la discusión investigación-creación en artes avances y propuestas. En: M. V. Casas (Ed.), Valoración de los procesos de creación artística y cultural en el marco de acreditación de programas. Bogotá: Ministerio de Educación, Consejo Nacional de Acreditación y Convenio Andrés Bello. CECAB publicaciones. ISBN 978-958-691-568-7 
Colciencias. (2015). Modelo de medición de grupos de investigación, desarrollo tecnológico o de innovación y de reconocimiento de investigadores del Sistema Nacional de Ciencia, Tecnología e Innovación, Año 2015 Convocatoria 737 de 2015. https://www.colciencias.gov.co/sistemasinformacion/modelo-medicion-grupos

Colciencias. Dirección de Fomento a la Investigación. (2016). Participación de la Comunidad de Artes, Arquitectura y Diseño en el Modelo en el Reconocimiento y Medición de Grupos de Investigación, Desarrollo Tecnológico o de Innovación y en el Reconocimiento de Investigadores del Sistema Nacional de Ciencia, Tecnología e Innovación 2014-2015.

Dasanti, M., Hulsijn; J. \& Van der Torre, L. (2000). Negotiation protocols and dialogue games. In Proceedings of the BNAIC, Site Ceer. Recuperado de http://citeseerx.ist.psu.edu/viewdoc/summary? doi=10.1.1.2.4363

De Bruyne P., Gielen P. (2011). Community art: the politics of trespassing. Amsterdam: Valiz. 2011, ISBN 9789078088509

Delgado, T. (2016). La creatividad y sus posibilidades. En: XXII Congreso Institucional de Investigaciones. Memorias, pp. 10-12. Universidad El Bosque. ISSN 2322-9047

Domínguez, R. (2015). Editorial. Iconofacto, 11 (17), 6-8. ISSN: 1900-2785 Recuperado de https://revistas.upb.edu.co/index.php/iconofacto/article/ view/6357/5959

EcuRed. Enciclopedia colaborativa de la red cubana. (Acceso: mayo 21 de 2017).

Fernández, M.B. y Johnson, D. (2015). Investigación-acción en formación de profesores: Desarrollo histórico, supuestos epistemológicos y diversidad metodológica. Psicoperspectivas, 14 (3), 93-105. Recuperado de https:// dx.doi.org/10.5027/psicoperspectivas-Vol14-Issue3-fulltext-626 
Findeli, A., Brouillet, D., Martin, S., Moineau, C. \& Tarrago, R. (2008). Research through Design and Transdisciplinarity: A Tentative Contribution to the Methodology of Design Research. En L. Léchot Hirt (Presidencia). "Focused"-Current design research projects and methods. Simposio llevado a cabo en la conferencia de la Swiss Design Network, Berna. https://hal.archives-ouvertes.fr/hal-00995468

Gómez, T.A. y Martínez P.O.S. (2014) Gestión del conocimiento como herramientaparala innovaciónyel cambioincrementalenlasorganizaciones. Desarrollo Gerencial, 6 (2), 153-179. Julio a diciembre 2014. Universidad Simón Bolívar - Barraquilla. ISSN: 2145-5147. Recuperado de http:// portal.unisimonbolivar.edu.co:82/rdigital/desarrollogerencial/index.php/ desarrollogerencial/issue/archive https://doi.org/10.17081/dege.6.2.471

Hernández, F. (2006). Campos, temas y metodologías para la investigación relacionada con las artes. En M. Gómez-Muntané, F. Hernández-Hernández y H.J. Pérez-López, Bases para un debate sobre investigación artística (pp. 9-50). Barcelona: Ministerio de Educación y Ciencia. I.S.B.N.: 84-369-43481 Recuperado en https://sede.educacion.gob.es/publiventa/bases-para-undebate-sobre-investigacion-artistica/investigacion-educativa/12147

Hernández, O. (2014). La creación y la investigación artística en instituciones colombianas de educación superior. En: A Contratiempo, (23). Recuperado de http://www.musigrafia.org/acontratiempo/?ediciones/revista-23.html

Leavy P. (2015). Method meets arts. Arts-based research practice. The Guilford Press, New York.

MIUR, AFAM (2017). Alta Formazione Artistica, Musicale e Coreutica - AFAM, Ministero dellÍstruzione e della Ricerca - MIUR, Italia. Disponible en http:// www.afam.miur.it/argomenti/personale-docente.aspx 
Nowotny, H., Scott, P. \& Gibbons, M. (2003). Introduction: 'Mode 2 ' revisited: The new production of knowledge. Minerva, 41(3), 179-194. https://www. jstor.org/stable/41821245

Osipov, V. (2013). The Wheel of Competition as a New Instrument of Strategic Management World. Applied Sciences Journal, 27 (8): 1083-1086, 2013. Disponible en SSRN: https://ssrn.com/abstract=2433728 DOI: 10.5829/ idosi.wasj.2013.27.08.13735.

Quintana, A. (2006). Metodología de investigación científica cualitativa. En: Quintana A. \& Montgomery W. (eds.). Psicología: tópicos de actualidad. Lima: UNMSM.

Scrivener, S. (2002). The art object does not embody a form of knowledge. Working papers in Art and Design, 2. Londres. Disponible en http://sitem. herts.ac.uk/artdes_research/papers/wpades/vol2/scrivenerfull.html

Universidad de Chile, (2013). Propuesta de Actualización de Criterios de MetaValoración Académica de la Creación Artística. Universidad de Chile.

Zuzek M., Talik M., Swierczynski T. et al. (2008) Formal model for contract negotiation in knowledge based virtual organizations. International Conference on Computational Science - ICCS - 2008. En: Lecture Notes in Computational Science. LNCS, 5103, 409-418. Disponible en https://link. springer.com/content/pdf/10.1007\%2F978-3-540-69389-5_47.pdf https:// doi.org/10.1007/978-3-540-69389-5_47

Como citar: Bonilla, H.A., Cabanzo, F., Delgado, T., Hernández, O.A., Niño, A.S. y Salamanca, J. (2019). Investigación-creación en Colombia: la formulación del "nuevo" modelo de medición para la producción intelectual en artes, arquitectura y diseño. Revista KEPES, 16 (20), 673-704. DOI: 10.17151/kepes.2019.16.20.24 